\title{
Quel soutien la SCPH apporte-t-elle aux pharmaciens d'hopitaux?
}

\author{
par Patricia Macgregor
}

$\mathrm{D}$ ans cette ère de changements, il importe de plus en plus que les membres de notre profession de pharmacien se serrent les coudes afin de réaliser notre vision collective de services de pharmacie axés sur le patient viables. Notre capacité de démontrer que nous avons une influence positive et mesurable sur les résultats thérapeutiques est un défi continu et les enjeux liés à l'accessibilité aux médicaments et à leur sécurité sont nombreux. Quelle est la valeur de la SCPH pour ses membres et notre profession en termes de soutien dans le présent ou pour l'avenir?

La Vision et les stratégies de la SCPH sont élaborées pour influencer l'avancement de la pratique de la pharmacie hospitalière, en outillant les pharmaciens pour le plein exercice de leurs compétences, en valorisant la profession, en collaborant avec les parties prenantes essentielles et en favorisant la mobilisation des membres et leur réseautage. La SCPH continue de s'engager auprès des gouvernements, des agences de réglementation et des associations professionnelles, comme en témoigne d'ailleurs ses interventions récentes sur deux graves difficultés : les pénuries de médicaments et l'incident de sous-dosage en oncologie survenu en Ontario et au Nouveau-Brunswick (voir le rapport d'examen officiel au www.health.gov.on.ca/fr/public/programs/cancer/ drugsupply/docs/report_thiessen_oncology_under-dosing.pdf).

Consciente du temps limité dont disposent les membres de la SCPH pour développer de nouveaux programmes, dossiers de décision et outils de gestion du changement, la Société a maintenant lancé trois trousses d'outils appuyant le projet SCPH 2015, chacune liée à un objectif particulier du projet. Une quatrième est à l'étude, pour aider les gestionnaires de pharmacies à obtenir le soutien de la haute direction pour les activités liées au projet SCPH 2015. En réponse aux souhaits des membres d'avoir facilement accès à des ressources éducatives et électroniques, la $\mathrm{SCPH}$ a proposé six webminaires éducatifs depuis décembre 2012, couvrant des sujets aussi divers que la pratique fondée sur des données probantes et les nouvelles normes et exigences d'Agrément Canada. Les membres peuvent écouter les enregistrements de ces webinaires à partir du site Web de la $\mathrm{SCPH}$. Les options de médias sociaux ne se limitent plus à Facebook et comprennent maintenant le blogue SCPH 2015 (cshp2015blog.com) et le compte Twitter (@cshp2015). Des affiches portant sur divers sujets, comme le bilan comparatif des médicaments et les indicateurs clés de performance clinique, qui ont été choisies lors de leur présentation à des conférences de la $\mathrm{SCPH}$, sont disponibles en format virtuel dans le site Web. Le concours 2013-2014 pour les pharmaciens étudiants comprendra des options pour souligner la portée éventuelle du projet SCPH 2015 sur les soins aux patients, notamment grâce à des affiches et à de courts récits, de même qu’à des vidéos. Juste à temps pour le mois de la sensibilisation à la pharmacie de 2013, la SCPH a diffusé une vidéo démontrant la valeur des pharmaciens d'hôpitaux que ses membres peuvent télécharger à partir de son site Web.

Depuis 2007, la SCPH travaille de concert avec le comité de rédaction du Rapport sur les pharmacies hospitalières canadiennes afin de mesurer les progrès quant à la mise en œuvre du projet SCPH 2015 (www.lillyhospitalsurvey.ca). Les mises à jour sur l'état d'avancement du projet SCPH 2015, qui viennent juste d'être lancées, sont des tableaux de bord pratiques pour rendre compte et comparer dans quelle mesure les objectifs du projet ont été atteints aux niveaux national et régional, et de son propre établissement (www.cshp.ca/programs/cshp2015/ index_e.asp).

Les membres peuvent aussi examiner les toutes dernières publications officielles ("Essais cliniques : Lignes directrices pour les pharmacies des établissement de santé » nouvellement mises à jour et « Documentation des activités des pharmaciens dans les dossiers de santé : Lignes directrices »), de même que le tout dernier cours d'éducation continue en ligne « Réduction du risque de contamination en pharmacie d'oncologie ».

Voilà un aperçu de la manière dont la SCPH apporte son soutien aux pharmaciens d'hôpitaux. La Société continuera de forger des alliances stratégiques et d'assurer sa participation en tant qu'acteur clé valorisant la pharmacie auprès des gouvernements, des agences de réglementation et d'autres associations professionnelles nationales, nord-américaines et internationales.

[Traduction par l'éditeur]

Patricia Macgregor, B. Sc., R. Ph., M. R. Pharm. S., M. H. Sc., C.H.E., est présidente et agente de liaison pour la vision de la Société canadienne des pharmaciens d'hôpitaux. 